\title{
Design of Hexagonal Shaped Split Ring Resonator for Multi-Resonant Behaviour
}

\author{
M. Jagadish and A.S. Pradeep
}

\begin{abstract}
In this paper, hexagonal shaped Split Ring Resonator (SRR) printed on Rogers's substrate is presented. Multi resonant behavior in microwave regime is obtained due to distinct magnetic resonances determined by number of concentric rings in the unit cell. ANSYS HFSS tool is used to model hexagonal shaped split ring resonator and plot its $S$ parameter for a range of frequency sweep. Electromagnetic parameters are retrieved to demonstrate the presence of negative permeability bandwidths.
\end{abstract}

Keywords--- Metamaterials, Negative Permeability.

\section{INTRODUCTION}

$M^{-}$ ETAMATERIALS are attracting more and more research interest for microwave and optical applications. Metamaterials are defined as the materials which have the ability to exhibit electromagnetic characteristics not readily found in naturally occurring materials [1]. Split Ring Resonators (SRR) are common in the metamaterial design as they exhibit negative permittivity and permeability close to their resonant frequencies [2]. A variety of SRR structures square, circular, triangular and elliptical have been conceived and studied in the literature available [3-8]. In this paper, three ring hexagonal shaped SRR is designed and simulated using HFSS to obtain multi-resonant behavior. Electromagnetic parameters are retrieved to demonstrate the presence of negative permeability bandwidths.

\section{Split Ring Resonator Design AND SimUlation RESULTS}

\section{A. Single Ring SRR}

At first, single ring hexagonal shaped SRR with varying side length is designed. The side length chosen are $3 \mathrm{~mm}$, 2mm, $1 \mathrm{~mm}$ for the SRR-1, SRR-2, and SRR-3 respectively as shown in figure 1 . All designed values are chosen for qualitative agreement with results for square SRR presented in [6].

M. Jagadish, Assistant Professor, Department of Electronics \& Communication Engineering, PESITM, Shivamogga, Karnataka, India. E-mail:jagadishm21@gmail.com

A.S. Pradeep Assistant Professor, Department of Electronics \& Communication Engineering, GEC, Hoovinahadagali, Karnataka. E-mail: pradeepas17@gmail.com

DOI: 10.9756/BIJRCE.8193

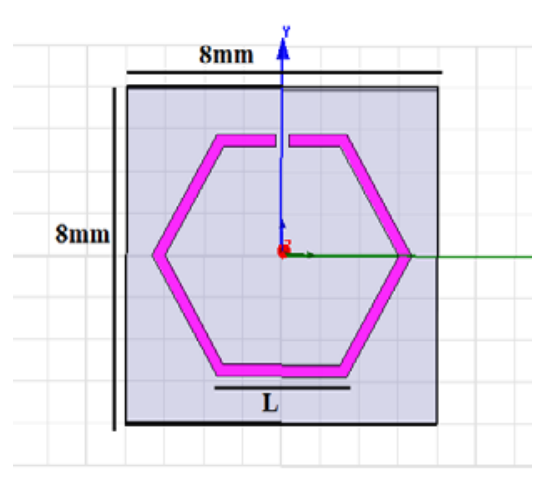

(a) SRR-1

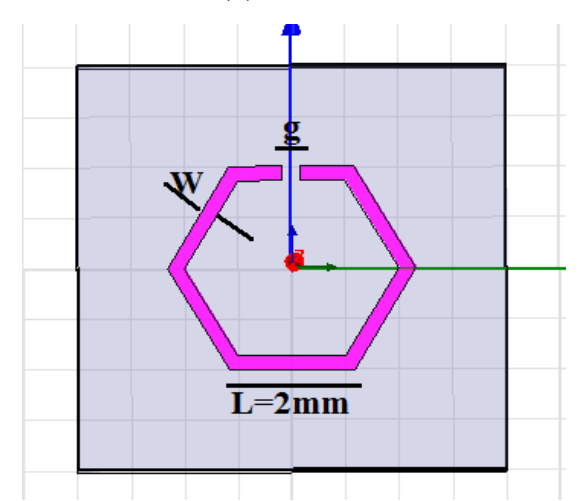

(b) SRR-2

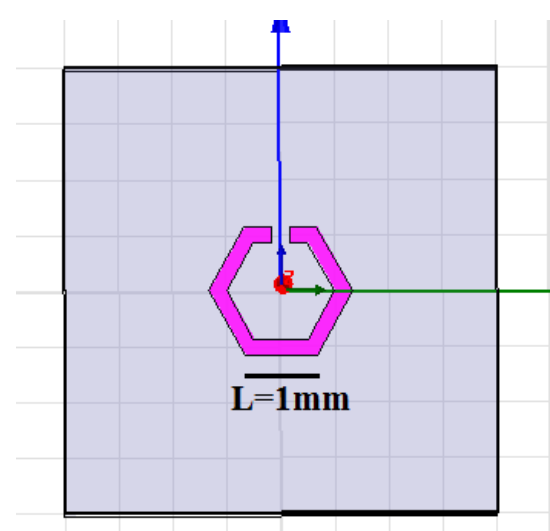

(c) SRR-3

Figure 1: Single Ring Hexagonal Shaped SRR with different $\mathrm{L}, \mathrm{w}=\mathrm{g}=0.33 \mathrm{~mm}$

The structure consists of metal ring printed with dielectric substrate (RO4003) with relative permittivity of 3.55 . Substrate dimensions are $8 \mathrm{~mm} \times 8 \mathrm{~mm} \times 0.81 \mathrm{~mm}$. The gap width $(\mathrm{g})$ is $0.33 \mathrm{~mm}$ and the width $(\mathrm{w})$ of the metal rings is $0.33 \mathrm{~mm}$. 
The return loss is the primary parameter in the analysis of SRR. The amplitude (in $\mathrm{dB}$ ) of S21 parameter for the single ring unit cell - SRR-1, SRR-2, SRR-3 is as shown in figure 2

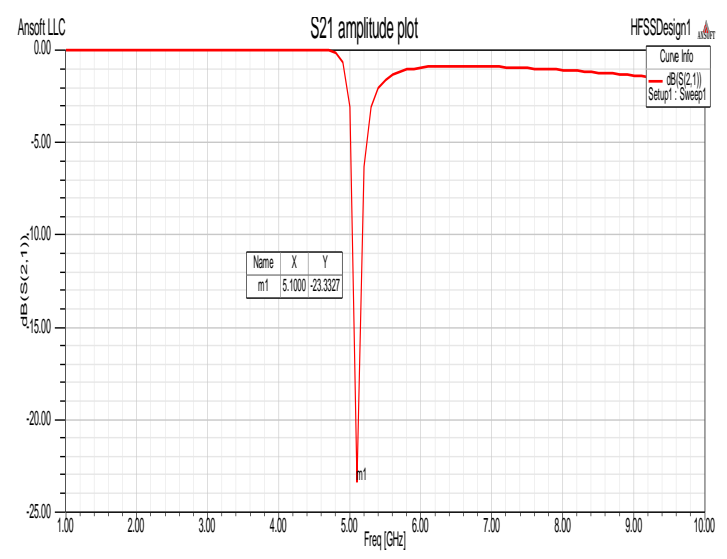

(a) Magnitude of S21 for SRR-1

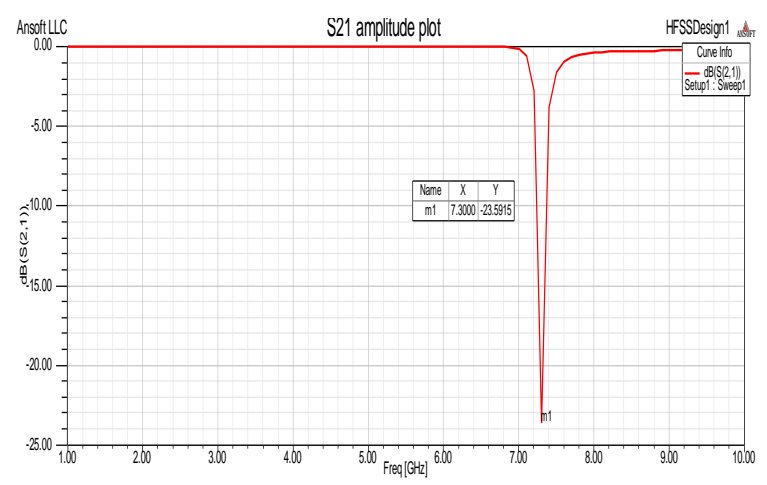

(b) Magnitude of S21 for SRR-2

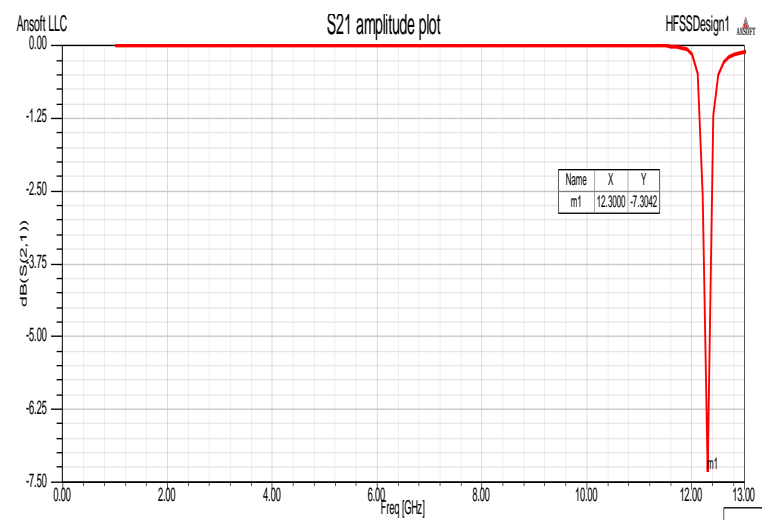

(C) Magnitude of S21 for SRR-3

Figure 2: Magnitude of S21 for Single Ring SRR

As seen in figure 2 the resonant frequencies for SRR-1, SRR-2, SRR-3 with side lengths of $1 \mathrm{~mm}, 2 \mathrm{~mm}$, and $3 \mathrm{~mm}$ occurs at $5.1 \mathrm{GHz}, 7.3 \mathrm{GHz}$ and $12.3 \mathrm{GHz}$ respectively. As the side length decreases (from $3 \mathrm{~mm}$ to $1 \mathrm{~mm}$ ) the resonant frequency shifts from lower to higher frequency $(5 \mathrm{~Hz}$ to $10 \mathrm{GHz}$ ) due to decrease in capacitance. Thus, one can obtain the desired frequency shift by tuning the side length of the SRR.

\section{B. Multi Ring SRR}

Later to obtain two resonant frequency two rings hexagonal shaped SRR is designed by combining SRR-1 and SRR-2 as shown in figure 3

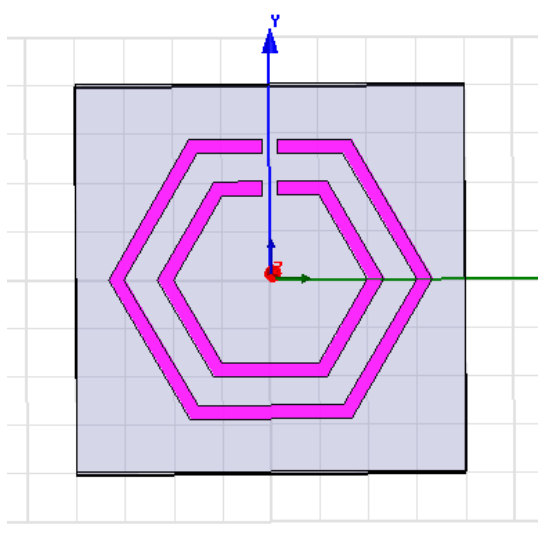

Figure 3: Hexagonal SRR with two Concentric rings Simulated Using HFSS

Similarly to obtain three resonant frequency three rings hexagonal shaped SRR is designed by combining SRR-1, SRR-2 and SRR-3 as illustrated in figure 4

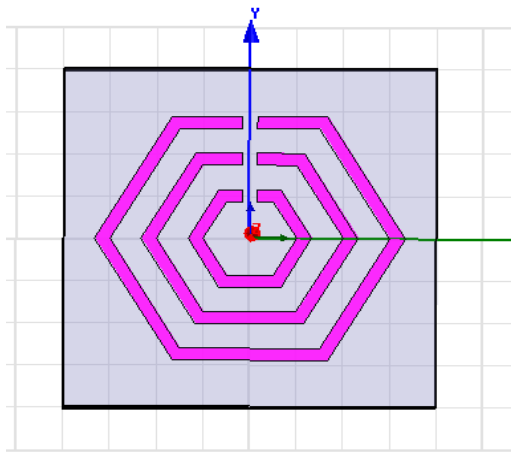

Figure 4: Hexagonal SRR with three Concentric Rings Simulated Using HFSS

The magnitude spectra of S21 parameter of two rings and three rings hexagonal shaped SRR are illustrated in figure $5 \mathrm{a}$ and $5 b$ respectively.

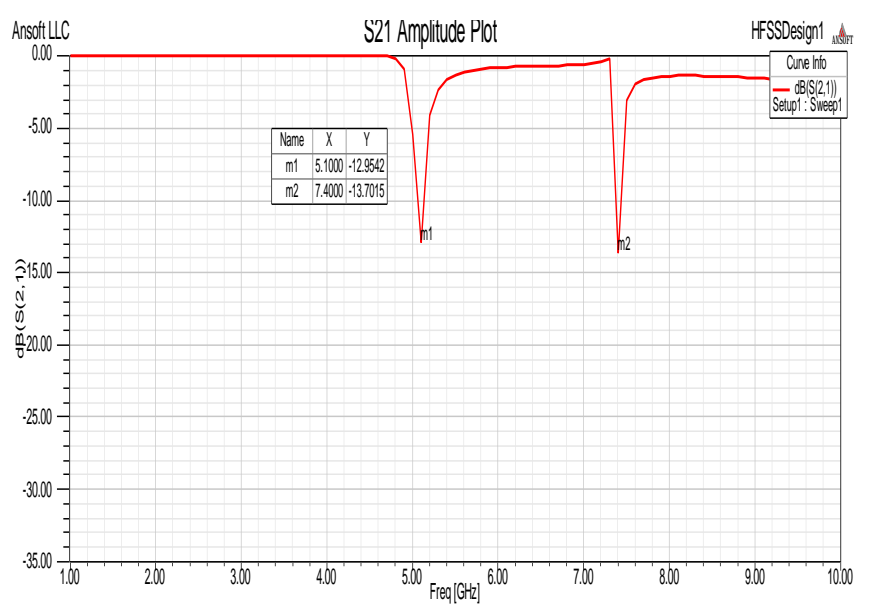

(a) Magnitude of S21 for SRR with two Rings 


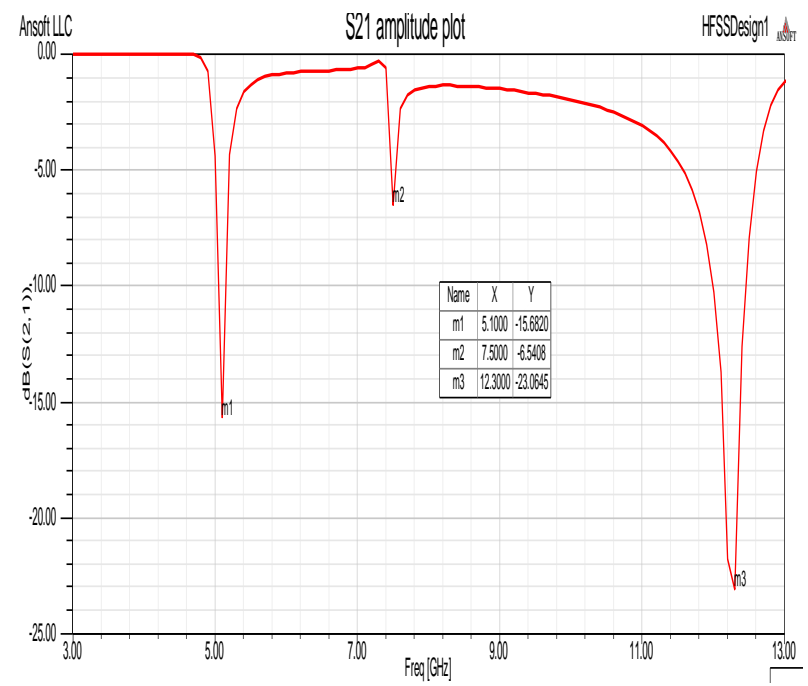

(b) Magnitude of S21 for SRR with Three Rings

Figure 5: Magnitude of S21 for SRR with Multiple Concentric Rings

As seen in figure 5 it is clear that SRR with two rings resonates at two different frequencies $-5.1 \mathrm{GHz}$ and $7.4 \mathrm{GHz}$

$$
n=\frac{1}{k_{0} d}\left[\left\{\left[\ln \left(e^{i n k_{0} d}\right)\right]\right.\right.
$$

$$
\begin{aligned}
& \varepsilon=\frac{n}{Z} \\
& \mu=n Z
\end{aligned}
$$

Wherek $_{0}$ is the wave vector.

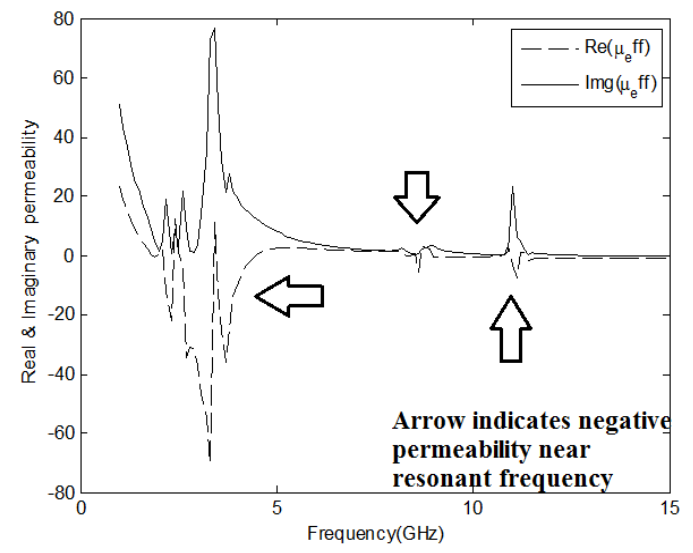

Figure 6: Real and Imaginary part of Permeability for SRR with three Rings

Figure 6 depicts that hexagonal shaped SRR with three rings has three bands with negative real effective permeability from 3.4 to $4.1 \mathrm{GHz}$, from 8.5 to $8.6 \mathrm{GHz}$, and from 10.9 to $11.1 \mathrm{GHz}$. whereas SRR with three rings resonates at three different frequencies - 5.1GHz, 7.5GHz, and $12.3 \mathrm{GHz}$. Thus multi band operation is achieved.

The resonant frequencies of all hexagonal shaped structure are summarized in Table. 1

Table 1: Summary of Simulation Results

\begin{tabular}{|l|c|}
\hline \multicolumn{1}{|c|}{ Structure } & Resonant Frequency in $\mathrm{GHz}$ \\
\hline SRR-1 & 5.1 \\
\hline SRR-2 & 7.3 \\
\hline SRR-3 & 12.3 \\
\hline Two Rings & $5.1 \& 7.4$ \\
\hline Three Rings & $5.1,7.5,12.3$ \\
\hline
\end{tabular}

From Table 1 it is depicted that the desired resonant frequency can be realized by tuning the side length of SRR. Also, the desired number of magnetic resonances can be realized by selecting the number of concentric ring within the limits of geometrical constraints.

\section{Electromagnetic Parameter Extraction}

The electromagnetic parameters such as effective permittivity $(\varepsilon)$, effective permeability $(\varepsilon)$ and refractive index (n) of hexagonal shaped SRR are extracted using the formula given in [9]
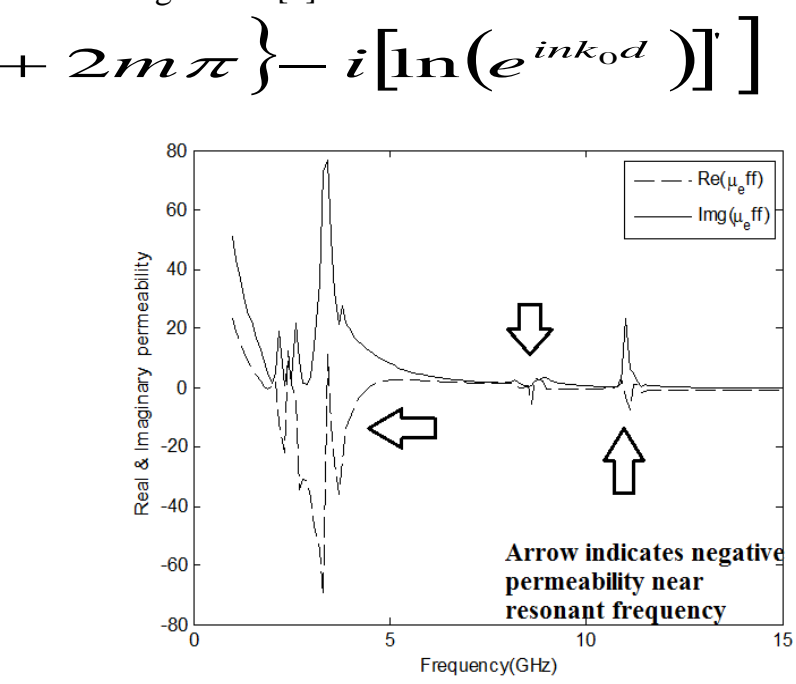

Figure 7: Real and Imaginary part of Permeability for SRR with three Rings

Figure 7 depicts that hexagonal shaped SRR with three rings has three bands with negative real effective permeability from 3.4 to $4.1 \mathrm{GHz}$, from 8.5 to $8.6 \mathrm{GHz}$, and from 10.9 to $11.1 \mathrm{GHz}$.

\section{CONCLUSION AND FUTURE ENHANCEMENT}

In this paper, hexagonal shaped SRR is designed to obtain multiple resonances within the microwave frequency range by selecting the number of concentric rings. It is showed by simulation using HFSS that the desired frequency can be realized by tuning the side length of SRR. The parameter extraction is used to demonstrate thepresence of negative permeability bandwidth. The designed hexagonal shaped SRR can be good candidate for metamaterial application in various portions of electromagnetic spectrum, especially in miniaturization of devices. 
In future, the effect of other SRR design parameters such as split gap width (g), ring width (w) on resonant frequency can be studied.

\section{REFERENCES}

[1] V.G. Veselago, "The electrodynamics of substances with simultaneously negative values of and?." Soviet Physics Uspekhi, Vol.10, No.4, Pp.509, 1968.

[2] C.T. Jun, D.R. Smith and R. Liu, "Metamaterials: Theory, Design, and Applications”, Springer, New York, 2010.

[3] C. Saha, J. Y. Siddiqui, Y.M.M. Antar, "Theoretical investigation of the square split ring resonator", Proceedings of URSI NA Radio Science Meet, 2007.

[4] C. Saha, J.Y. Siddiqui, "Estimation of the resonance frequency of conventional \& rotational circular split ring resonators”, IEEEApplied Electromagnetics Conference (AEMC), Kolkata, 2009.

[5] M.R. Vidyalakshmi and Dr.S. Raghavan, "A CAD Model of Triangular Split Ring Resonator Based on Equivalent Circuit Approach,” IEEE Applied Electromagnetics Conference (AEMC), Kolkata, Pp.1-4, 2009.

[6] V. Sharma, S.S. Pattnaik, T. Garg and S. Devi, "A microstrip metamaterial split ring resonator", International Journal of Physical Sciences, Vol.6, No.4, Pp.660-663, 2011.

[7] R. Marquez, F. Mesa, J. Martel, F. Medina, "Comparative analysis of edge- and broadside-coupled split ring resonators formetamaterial design-theory and experiments", IEEE Transactions on Antennas Propagation, Vol.51, No.10, pp.2572-2581, 2003.

[8] V.J. Bashenoff, "Abbreviated methods for calculating the inductance of irregular plane polygons of round wire", Proceedings ofInstitute of Radio Engineers, Vol.15, No.12, Pp.1013-1039, 1927.

[9] Q. Li and A.P. Feresidis, "Reduction of mutual coupling between compact MIMO antennas arrays", In Antennas and Propagation Conference (LAPC), 2010 Loughborough, Pp. 277-280, 2010.

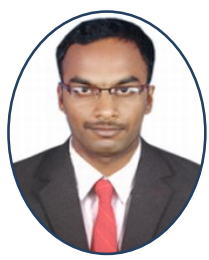

M. Jagadish received the BE degree in Electronics and Communication Engineering from SVCE, Bangalore, Karnataka and M.tech degree in Communication Systems from SRM University, Chennai, Tamil Nadu in 2012 and 2014 respectively. Since 2015 he has been an Assistant Professor in the Department of ECE at PESITM, Shivamogga. His research interest include antenna system design and signal processing.

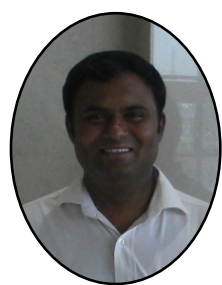

A.S. Pradeep received the BE degree in Electronics and Communication Engineering from UBDT Engineering College, Davanagere, Karnataka and M.tech degree in Electronics from Sir MVIT, Bangalore, Karnataka in 2003 and 2006 respectively. Currently he is persuingPh.D degree from Visvesvaraya Technological University, Belagavi, Karnataka under the guidance of Dr. G. A. Bidkar.He worked as an Assistant Professor in the Department of ECE at RVCE, Bangalore during 20072010. He he is working as an Assistant Professor in the Department of ECE at GEC, Hoovinahadagali, since 2010. His research interest include microwave and antenna design. 